\title{
SÍNDROME DE BROWN-SÉQUARD POR HÉRNIA DISCAL CERVICAL A DUPLO NÍVEL. CASO CLÍNICO E REVISÃO DA LITERATURA
}

\author{
BROWN-SÉQUARD SYNDROME BY DOUBLE LEVEL CERVICAL DISC HERNIATION. \\ CASE REPORT AND REVIEW OF THE LITERATURE
}

\author{
SÍNDROME DE BROWN-SÉQUARD POR HERNIA DISCAL CERVICAL EN NIVEL DOBLE. \\ CASO CLIINICO Y REVISIÓN DE LA LITERATURA
}

Jorge Miguel Silva Ribeiro Olliveira Alves ${ }^{1}$, Paulo Peixoto ${ }^{1}$, Nuno Ferreira ${ }^{1}$, Rui Martins ${ }^{1}$, João Correia ${ }^{1}$ Fernando Silva ${ }^{2}$, Carlos Sousa ${ }^{3}$

\begin{abstract}
RESUMO
Descrição do caso clínico de um paciente com síndrome de Brown-Séquard por hérnia de disco cervical em duplo nível, 40 anos, do sexo masculino, sem história de patologia prévia da coluna cervical; teve início insidioso de paresia nos membros direitos, associada à diminuição da sensibilidade dolorosa e térmica do hemicorpo esquerdo, após mergulho que ocasionou trauma indireto da coluna cervical. A RM da coluna cervical mostrou hérnias discais paramedianas direitas nos níveis C4-C5 e C5-C6 com compressão da metade direita do cordão medular e hiperintensidade em T2. Foi realizada descompressão por via anterior e artrodese dos níveis afetados. 0 paciente recuperou-se parcialmente dos déficits neurológicos, após um longo programa de reabilitação funcional. O tratamento cirúrgico e um programa de reabilitação precoce são da maior importância para a recuperação neurológica de paciente com síndrome de Brown-Séquard por hérnia de disco cervical.
\end{abstract}

Descritores: Deslocamento do disco intervertebral; Síndrome de Brown-Séquard; Artrodese; Vértebras cervicais.

\begin{abstract}
This article presents a case in which Brown-Séquard syndrome resulted from a double level cervical disc herniation. A 40-year-old man without previous history of cervical pathology, presented with insidious right arm and leg paresis associated with associated with decreased pain and thermal sensitivity in the left hemibody after diving which caused indirect trauma of the cervical spine. Magnetic resonance imaging of the cervical spine showed double level disc herniation in C4-C5 and C5-6 with compression of the right half of spinal cord and hyperintensity in T2-weighted images. After a complete decompression of neural structures, a double level interbody fusion was performed. There was partial recovery of neurological status after a long period of physical therapy. Early surgical intervention and prompt rehabilitation are paramount to achieve neurological recovery in patients with Brown-Séquard syndrome resulting from a cervical disc herniation.
\end{abstract}

Keywords: Intervertebral disc displacement; Brown-Séquard syndrome; Arthrodesis; Cervical vertebrae.

\section{RESUMEN}

Descripción del caso clínico de un paciente con síndrome de Brown-Séquard por hernia de disco cervical en nivel doble, 40 años, del sexo masculino, sin historial de patología previa de la columna cervical; tuvo inicio insidioso de paresia en los miembros derechos, vinculada con la disminución de la sensibilidad dolorosa y térmica del hemicuerpo izquierdo, después de zambullida que ocasionó traumatismo indirecto de la columna vertebral. La RM de la columna cervical mostró hernias discales paramedianas derechas, en los niveles C4-C5 y C5-C6, con compresión de la mitad derecha del cordón medular e hiperintensidad en T2. Se realizó descompresión por vía anterior y artrodesis de los niveles afectados. El paciente se recuperó, parcialmente, de los déficits neurológicos, después de un programa prolongado de rehabilitación funcional. El tratamiento quirúrgico y un programa de rehabilitación precoz son de máxima importancia para la recuperación neurológica del paciente con síndrome de Brown-Séquard por hernia de disco cervical.

Descriptores: Desplazamiento del disco intervertebral; Síndrome de Brown-Séquard; Artrodesis; Vértebras cervicales.

\section{INTRODUÇÃO}

A Síndrome de Brown-Séquard é uma entidade classicamente encontrada nas lesões traumáticas da coluna. O quadro clínico reflete uma hemissecção do cordão medular, com perda de função motora ipsilateral associada a um déficits da sensibilidade dolorosa e térmica contra-lateral ${ }^{1}$. As causas mais frequentes da síndrome são as lesões traumáticas ou tumorais (metastáticas ou intrínsecas). No entanto, estão descritas outras etiologias como a isquemia do cordão medular; patologia infecciosa ou inflamatória incluindo esclerose múltipla; hemorragia incluindo hematomielia e hematoma sub ou epidural; e doença degenerativa². A hérnia discal cervical é uma causa pouco frequente da síndrome ${ }^{3}$. Os autores reportam o caso clínico de um paciente com hérnia discal cervical a duplo nível que provocou hemicompressão severa do cordão medular resultando em Síndrome de Brown-Séquard.

1. Interno Complementar de Ortopedia Serviço de Ortopedia eTraumatologia do Centro Hospitalar doTâmega e Sousa, Porto, Portugal.

2. Assistente Hospitalar de Ortopedia Serviço de Ortopedia eTraumatologia do Centro Hospitalar do Tâmega e Sousa, Porto, Portugal.

3. Chefe de Serviço de Ortopedia Serviço de Ortopedia eTraumatologia do Centro Hospitalar do Tâmega e Sousa, Porto, Portugal.

Trabalho realizado no Serviço de Ortopedia eTraumatologia do Centro Hospitalar doTâmega e Sousa, Porto, Portugal. 


\section{RELATO DO CASO}

Paciente de 40 anos, do sexo masculino, sem história de patologia da coluna cervical, teve inicio insidioso de hemiparesia direita e alteração da sensibilidade do hemicorpo à esquerda após mergulho com trauma indirecto da coluna cervical.

O paciente apresentava diminuição das mobilidades da coluna cervical. No exame neurológico havia diminuição da sensibilidade dolorosa e térmica nos membros superior e inferior esquerdos. No membro superior direito foi identificada uma área hiperalgica correspondente aos territórios de C5 e C6. O reflexo bicipital e braquiorradial estavam diminuídos do lado direito. Nos membros inferiores foi encontrada hiperreflexia, sobretudo à direita. No exame motor havia diminuição da força muscular dos membros superior e inferior direito (3/5). O quadro clínico era consistente com Síndrome de Brown-Sèquard.

A RMN da coluna cervical (Figura 1) mostrou duas hérnias discais paramedianas direitas nos níveis C4-C5 e C5-C6 com hemicompressão do cordão medular. As imagens em T2 mostram áreas de hipersinal medular direito.

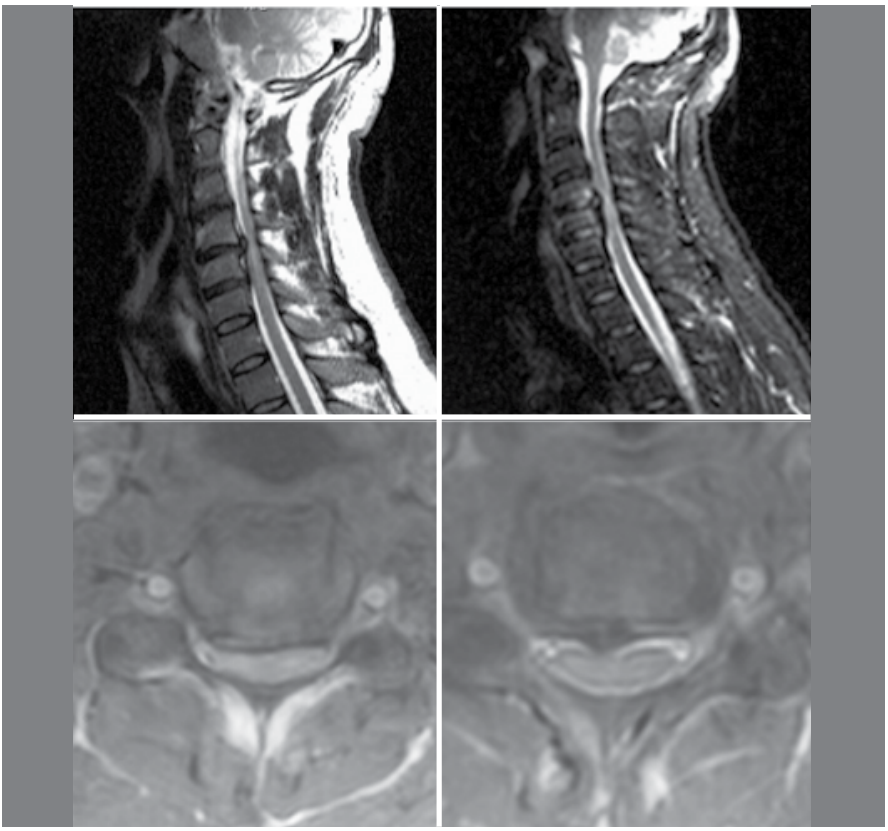

Figura 1. Compressão do cordão medular e hiperssinal em T2 ao nível dos espaços C4-C5 e C5-C6.
Foi utilizada uma abordagem cervical anterior standard através da qual se realizou a descompressão das estruturas neurológicas. Foi encontrada uma grande quantidade de material discal herniada posteriormente, comprimindo a metade direita do cordão medular nos espaços C4-C5 e C5-C6. Após descompressão completa das estruturas neurológicas, foi realizada artrodese interssomática a dois níveis com cage PEEK contendo matriz óssea desmineralizada. Foi utilizada fluoroscopia intra-operatória para verificação do correcto posicionamento do implante. O paciente manteve imobilização com colar cervical mole durante 12 semanas.

O pós-operatório decorreu sem intercorrências. O paciente Iniciou reabilitação no $2^{\circ}$ dia após a cirurgia tendo recuperado parcialmente dos déficits neurológicos após um longo programa de reabilitação funcional, que continua (sete meses de evolução).

\section{DISCUSSÃO}

A hérnia discal cervical é uma causa pouco frequente (cerca de 2,6\% dos casos) de síndrome de Brown-Séquard ${ }^{4}$. O primeiro autor a descrever a associação foi Stookey ${ }^{5}$, em 1928, com a publicação de três casos clínicos. Após a primeira publicação foram

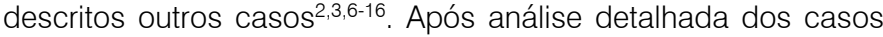
descritos verificou-se que a idade média de aparecimento rondou os 40A (25-73A) e houve um ligeiro predomínio do sexo masculino. Os níveis mais frequentemente envolvidos foram C4-C5 e C5-C6, e à semelhança deste caso só em dois paciente que estiveram envolvidos em 2 níveis ${ }^{11,16}$. O material herniado pode ter uma localização intra-dural, o que não aconteceu neste caso. O intervalo entre o início dos sintomas e o diagnóstico pode ser elevado (18 meses em dois pacientes). Só em dois casos houve trauma relevante envolvido ${ }^{7,8}$, e embora o presente caso fosse de apresentação insidiosa, o trauma cervical após o mergulho pareceu ser o mecanismo desencadeador da situação. A RMN veio facilitar tanto o diagnóstico como a localização precisa da hérnia discal (mediana, mediolateral ou lateral), auxiliando o planeamento cirúrgico ${ }^{14}$. Todos os fpaciente foram tratados cirurgicamente e à semelhança deste caso a descompressão e artrodese por via anterior foi a técnica mais utilizada. A recuperação total dos déficits neurológicos só foi conseguida em seis casos ${ }^{2,3}$. Com 7 meses de follow-up o nosso paciente se recuperou parcialmente dos seus déficits.

\section{RECOMENDAÇÕES}

A Síndrome de Brown-Séquard provocada por hérnia discal cervical a duplo nível é uma entidade rara. Intervenção cirúrgica e reabilitação precoces são recomendadas para atingir os melhores resultados.

\section{REFERÊNCIAS}

1. Leventhal ML. Fractures, dislocations, and fracture dislocations of spine. In. Canale ST, editor. Campbell's operative orthopaedics. 9th ed. Philadelphia: Mosby/Elsevier; 1998. p. 2709

2. Mastronardi L, Ruggeri A. Cervical disc herniation producing Brown-Sequard syndrome: case report. Spine (Phila Pa 1976). 2004;29(2):E28-31.

3. Sayer FT, Vitali AM, Low HL, Paquette S, Honey CR. Brown-Sèquard syndrome produced by $\mathrm{C} 3-\mathrm{C} 4$ cervical disc herniation: a case report and review of the literature. Spine (Phila Pa 1976). 2008;33(9):E279-82

4. Jomin M, Lesoin F, Lozes G, Thomas CE 3rd, Rousseaux M, Clarisse J. Herniated cervical discs. Analysis of a series of 230 cases. Acta Neurochir (Wien). 1986;79(2-4):107-13.

5. Stookey B. Compression of the spinal cord due to ventral extradural cervical chondromas: diagnosis and surgical treatment. Arch Neurol Psychiatry. 1928;20:275-91.

6. Antich PA, Sanjuan AC, Girvent FM, Simó JD. High cervical disc herniation and BrownSequard syndrome. A case report and review of the literature. J Bone Joint Surg Br. 1999;81(3):462-3.

7. Börm W, Bohnstedt T. Intradural cervical disc herniation. Case report and review of the literature. J Neurosurg. 2000;92(2 Suppl):221-4.

8. Clatterbuck RE, Belzberg AJ, Ducker TB. Intradural cervical disc herniation and Brown-

Séquard's syndrome. Report of three cases and review of the literature. J Neurosurg 2000;92(Suppl 2):236-40.

9. Dürig M, Zdrojewski B. [Intrathecal herniation of a cervical disc. A case report (author's transl)]. Arch Orthop Unfallchir. 1977:87(2):151-7.

10. Eisenberg RA, Bremer AM, Northup HM. Intradural herniated cervical disk: a case report and review of the literature. AJNR Am J Neuroradiol. 1986;7(3):492-4.

11. Kohno M, Takahashi $\mathrm{H}$, Yamakawa $\mathrm{K}$, Ide K, Segawa H. Postoperative prognosis of BrownSéquard-type myelopathy in patients with cervical lesions. Surg Neurol. 1999;51(3):241-6.

12. Roda JM, Gonzalez C, Blázquez MG, Alvarez MP, Argüello C. Intradural herniated cervical disc. Case report. J Neurosurg. 1982:57(2):278-80.

13. Rumana CS, Baskin DS. Brown-Sequard syndrome produced by cervical disc herniation: case report and literature review. Surg Neurol. 1996;45(4):359-61.

14. Schneider SJ, Grossman RG, Bryan RN. Magnetic resonance imaging of transdural herniation of a cervical disk. Surg Neurol. 1988;30(3):216-9.

15. Sprick C, Fegers S. [Intradural sequestration of cervical intervertebral disk displacement] Nervenarzt. 1991;62(2):133-5.

16. Finelli PF, Leopold N, Tarras S. Brown-Sequard syndrome and herniated cervical disc. Spine (Phila Pa 1976). 1992;17(5):598-600. 